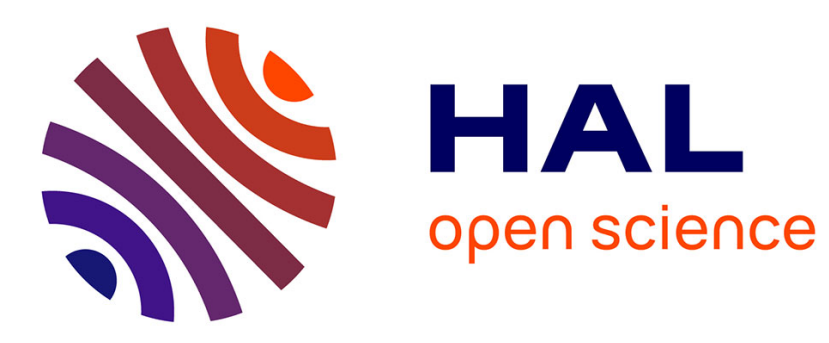

\title{
On the Influence of Phase Change in Highly Loaded Frictional Contacts
}

\author{
Serigne Ndiaye, Laetitia Martinie, David Philippon, Marjolaine Gonon-Caux, \\ Jeremie Margueritat, Philippe Vergne
}

\section{- To cite this version:}

Serigne Ndiaye, Laetitia Martinie, David Philippon, Marjolaine Gonon-Caux, Jeremie Margueritat, et al. On the Influence of Phase Change in Highly Loaded Frictional Contacts. Tribology Letters, 2020, 68 (2), pp.54. 10.1007/s11249-020-01298-6 . hal-02546946

\section{HAL Id: hal-02546946 \\ https://hal.science/hal-02546946}

Submitted on 22 Oct 2020

HAL is a multi-disciplinary open access archive for the deposit and dissemination of scientific research documents, whether they are published or not. The documents may come from teaching and research institutions in France or abroad, or from public or private research centers.
L'archive ouverte pluridisciplinaire HAL, est destinée au dépôt et à la diffusion de documents scientifiques de niveau recherche, publiés ou non, émanant des établissements d'enseignement et de recherche français ou étrangers, des laboratoires publics ou privés. 


\title{
On the influence of phase change in highly loaded frictional contacts
}

\author{
Serigne Ndiaye ${ }^{\mathrm{a}}$, Laetitia Martinie ${ }^{\mathrm{a}, 1}$, David Philippon ${ }^{\mathrm{a}}$, Marjolaine Gonon-Caux ${ }^{\mathrm{a}}$, Jérémie Margueritat ${ }^{\mathrm{b}}$, \\ Philippe Vergne ${ }^{a}$ \\ a Univ Lyon, INSA Lyon, CNRS, LaMCoS - UMR5259, F-69621 Villeurbanne, France \\ b Univ Lyon, Université Lyon1, CNRS, ILM - UMR5306, F-69622 Villeurbanne, France
}

DOI: $10.1007 / \mathrm{s} 11249-020-01298-6$

\begin{abstract}
Highly loaded lubricated contacts may present a friction plateau on which the friction force becomes independent from the sliding velocity. Although this phenomenon has been known for a long-time, its physical origin remains poorly known. The present paper aims at giving further insight on the physical mechanisms triggering the friction plateau. The study specifically focuses on the influence of pressure on the physical state of the lubricant. An uncoupled experimental approach (rheology, tribology, spectrometry) has been conducted on a model lubricant. The lubricant behavior has been probed both at rest by Brillouin light scattering spectroscopy and dynamically under stationary conditions in a lubricated contact. The first experimental approach led to evidence a transition pressure associated with the lubricant glass transition, which has then been correlated to the lubricant macroscopic response in friction. We show that the onset of the plateau behavior occurs for Hertzian pressures close to the glass transition pressure, corresponding to a very small fraction of lubricant in a "nominal glassy state" within the contact.
\end{abstract}

\section{Keywords}

Lubrication, friction, limiting shear stress, glass transition, Brillouin light scattering.

\section{Introduction}

Understanding the behavior of contacts submitted to extreme conditions is necessary to meet the growing need for miniaturizing the mechanisms and increasing their performances while preserving their sustainability.

If the influence of thermal effects is excluded, the resulting friction curves have been shown to exhibit three regimes in which the lubricant successively experiences a linear, a shear thinning and a plateau like behavior

${ }^{1}$ Corresponding author: laetitia.martinie@insa-lyon.fr 
for very low, low and medium slide-to-roll ratios $(S R R)$, respectively. The last regime is associated to the limiting shear stress (LSS) concept, which corresponds to a friction force that is no longer rate dependent. Up to now, although the linear and shear thinning regimes are fairly well numerically predicted, the third regime is still poorly understood, which leads to a severe limitation for friction prediction and even hampers a wise formulation of new lubricants. Moreover, it should be noted that the plateau regime occurs at high contact pressure, as can be found in most lubricated mechanisms such as rolling element bearings, gears, cam-tappet systems, etc. which represent an incalculable number of systems that should be made more efficient and more durable.

Previous researches intended to adjust friction models at high sliding speed by including thermal dissipation through a heat source term in the generalized Reynolds equation increasing as the square of the shear rate according to [1]. However, it has been shown that thermal effects alone cannot explain the friction plateau observed at high sliding speeds in highly loaded lubricated contacts [2-3].

Several friction scenarios have been put forward in the literature to explain how and why the plateau regime can occur [4]. Among them, the lubricant experiencing a glass transition [5-6] does make sense as this plateau behavior only occurs beyond a critical contact pressure [2]. However, in the works that support this scenario, no measurement of glass transition has been performed to confirm this assumption, neither simultaneously with friction measurement, nor out of the contact. Identifying precisely the glass transition remains indeed difficult because the physics of this transition in lubricants is not clear yet and far from being trivial to characterize. It is widely agreed that glass transition is of kinetic origin [7-9]. According to Ediger, it is "a kinetic event which depends upon the crossing of an experimental time scale and the time scales for molecular rearrangements". However, this definition does not account for the thermodynamic peculiarities that accompany this transition. Several definitions are used in the literature. Most of them rely on a temperature dependent material behavior which is characterized by the glass transition temperature, $T_{g}$. It is commonly determined as the (pressure-dependent) temperature at which the heat capacity (or thermal expansion coefficient) exhibits a steep change, or even more simply the temperature at which the sample volume evolution changes slope [10]. However, such thermodynamic data are rarely available for lubricants and more empirical criteria are practically used, as the temperature at which i) the relaxation time exceeds $100 \mathrm{~s}$ or ii) the dynamic viscosity exceeds $10^{12}$ Pa.s [7, 10-12]. Furthermore, $T_{g}$ is known to depend on the sample cooling rate, which further complicates any universal constitutive equation, even if this dependency can be relatively weak according to [7]. In addition, the identification of the glass transition temperature depends on the experimental technique. Typically, relaxation times measured upon vitrification range between 0.1 and $1000 \mathrm{~s}$ [10]. Such relaxation times (or high viscosities) lead to the general definition of the glass transition reported in the New York Times [13] "basically a rate of flow so slow that it is too boring and time-consuming to watch". 
From the dynamics point of view, when a liquid is cooled below its melting point or pressurized in an equivalent manner above its corresponding "melting pressure", and in both cases quickly enough to prevent crystallization, molecular motion slows down strongly due to the presence of neighbors that form a "cage" $[9,14-15]$ around each molecule. The time required for the latter to rearrange becomes significantly longer than any laboratory realistic timescale. Therefore, the liquid structure appears to be frozen, which explains the change in the expansion coefficient variation. This transition takes place over a certain range of temperature (or pressure), called the "transformation range". Furthermore, it has been shown that while cooling, molecules do not slow down uniformly [11, 16-17]. Spatial dynamical heterogeneities have been measured while, surprisingly, involving no microstructural heterogeneities [13]. Actually in glasses, dynamics is thought to emerge through cooperative motions, described as string-like motions in some works [16]. However, currently no general consensus has been found on the length scales of these heterogeneities [7, 17]. Furthermore, they are thought to persist at least as long as the ensemble average relaxation time, with a spatial distribution of local relaxation times spreading over several orders of magnitude [17].

Despite the lack of knowledge on the actual mechanisms behind these phase changes, the possible existence of a glass transition experienced by the lubricant in highly loaded contacts has been considered many times to explain the friction plateau. Indeed, the high pressures (> $1 \mathrm{GPa}$ ) and high shear rates endured by the lubricant leave only few chances for the molecules to rearrange.

In this work, both tribological measurements and determination of the glass transition have been conducted in the same temperature and pressure domains in order to correlate the friction plateau onset with the lubricant physical state. Indeed, this should help to identify the role of the state of the lubricant under friction and more specifically when the plateau regime is achieved to, in a further step, investigate the shear distribution in the lubricating film.

In the first part, results from rheological and thermo-physical experiments are reported. This leads us to derive a rheological model and to correlate the lubricant rheological behavior to its presumed physical state. In the second part, the specific experimental techniques used in this study, Brillouin spectroscopy on one hand and tribometry on the other hand, are briefly presented.

Then, we show in the third part that experimental results measured over a large range of pressure at different temperatures tend to strongly correlate the onset of the friction plateau with the phase transition experienced by the fluid. Although the transition experienced by lubricants between liquid and glassy states has already been characterized by Brillouin spectroscopy in literature (see III.1), it is the first time that it is directly confronted to friction measurements in a highly loaded contact.

Finally, these results are discussed with respect to the rheological response of the lubricant. Some insights on the physical mechanisms most likely to occur are proposed relatively to the specific conditions inside a contact. 


\section{Materials}

\section{II.1. Rheology}

Benzyl benzoate (BB) has been used as a model lubricant in this experimental study: it is the ester of benzyl alcohol and benzoic acid, of formula $\mathrm{C}_{6} \mathrm{H}_{5} \mathrm{COOCH}_{2} \mathrm{C}_{6} \mathrm{H}_{5}$. It was supplied by abcr $\mathrm{GmbH}$ (Karlsruhe, Germany), of $99 \%$ purity (CAS 120-51-4). The choice of this lubricant was motivated by, among different reasons, its response in spectroscopic measurements and its physical properties at ambient condition.

Its rheological characterization has been performed according to the methodology described in [18] and the results were summarized in [2]. A Vogel Tamman Fulcher (VTF) correlation [9] and an improved WLF-type model [19], respectively characterizing its viscosity temperature and viscosity-temperature-pressure dependencies were used. The latter model (referred to as the modified WLF in the following) is an improvement of the work of Yasutomi et al. [20], who had extended the Williams-Landel-Ferry timetemperature superposition principle to pressure. The improved model gives a more accurate representation of viscosity at both low and high pressure: the interested reader can refer to ref. [19] for more details. It writes:

$$
\begin{aligned}
& \eta(T, P)=\eta_{g} \exp \left[\ln 10 \frac{-C_{1}\left(T-T_{g}(P)\right) F(P)}{C_{2}+\left(T-T_{g}(P)\right) F(P)}\right] \\
& T_{g}(P)=T_{g}(0)+A_{1} \ln \left(1+A_{2} P\right) \\
& F(P)=\left(1+B_{1} P\right)^{B_{2}}
\end{aligned}
$$

Where $T_{g}(P)$ and $T_{g}(0)$ are the glass transition temperatures at pressure $P$ and atmospheric pressure, respectively; $\eta_{g}$ is the viscosity at the glass transition temperature and atmospheric pressure, $T_{g}(0) ; F(P)$ is the dimensionless relative thermal expansion of the free volume; $A_{1}, A_{2}, B_{1}, B_{2}, C_{1}$ and $C_{2}$ are the constants of the modified WLF model.

$T_{g}(0), A_{1}, A_{2}, B_{1}, B_{2}, C_{1}$ and $C_{2}$ were determined by least mean squares regressions to the rheological measurements performed in the following ranges: -10 to $+120^{\circ} \mathrm{C}$ (at ambient pressure, VTF correlation), 40 $80-130^{\circ} \mathrm{C}$ and up to $700 \mathrm{MPa}$ (modified WLF model). The values are reported in Table 1 together with $S D$, the standard deviation calculated on the absolute values of the relative deviations between predicted and measured viscosities.

To highlight the fluid sensitivity with temperature and pressure, the fluid viscosity is plotted versus both parameters in Figure 1, indicating its fragility compared to other fluids, used here as strong (i.e. with low fragility) or fragile (high fragility) reference liquids. This property has been defined and widely used in the literature with respect to temperature $[7,9-11,14]$. It has also more recently been defined relatively to pressure [21]. According to Angell [9] and as reported by Casalini and Roland [22], it refers to the loss of local 
structure (short range order) with increasing $T$ across the glass transition. For fragile liquids, this structure is rapidly disrupted and large changes in viscosity with $T_{g} / T$ are observed. Conversely, strong fluids can keep their short range order to higher temperatures, with consequently relatively narrow viscosity variations [22]. These two distinct behaviors are illustrated in Figure 1 left (temperature sensitivity) for o-terphenyl and Si02 (results from [14]), and in Figure 1 right (pressure sensitivity) for a PAO (polyalphaolefin) and DBEB (dibenzylethylbenzene) (results from [21]).

\begin{tabular}{|c|c|}
\hline$C_{1}$ & 16.00 \\
\hline$C_{2}\left({ }^{\circ} \mathrm{C}\right)$ & 14.55 \\
\hline$A_{1}$ & 400.1896 \\
\hline$A_{2}\left(\mathrm{MPa}^{-1}\right)$ & 0.000363 \\
\hline$B_{1}\left(\mathrm{MPa}^{-1}\right)$ & 0.005391 \\
\hline$B_{2}$ & -0.3989 \\
\hline$T_{g}(0)\left({ }^{\circ} \mathrm{C}\right)$ & -81.7343 \\
\hline$\eta_{g}(\mathrm{~Pa} . \mathrm{s})$ & $1.010^{+12}$ \\
\hline$S D$ & $8.24 \%$ \\
\hline
\end{tabular}

Table 1: constants of the modified WLF model applied to rheological measurements performed on benzyl benzoate.
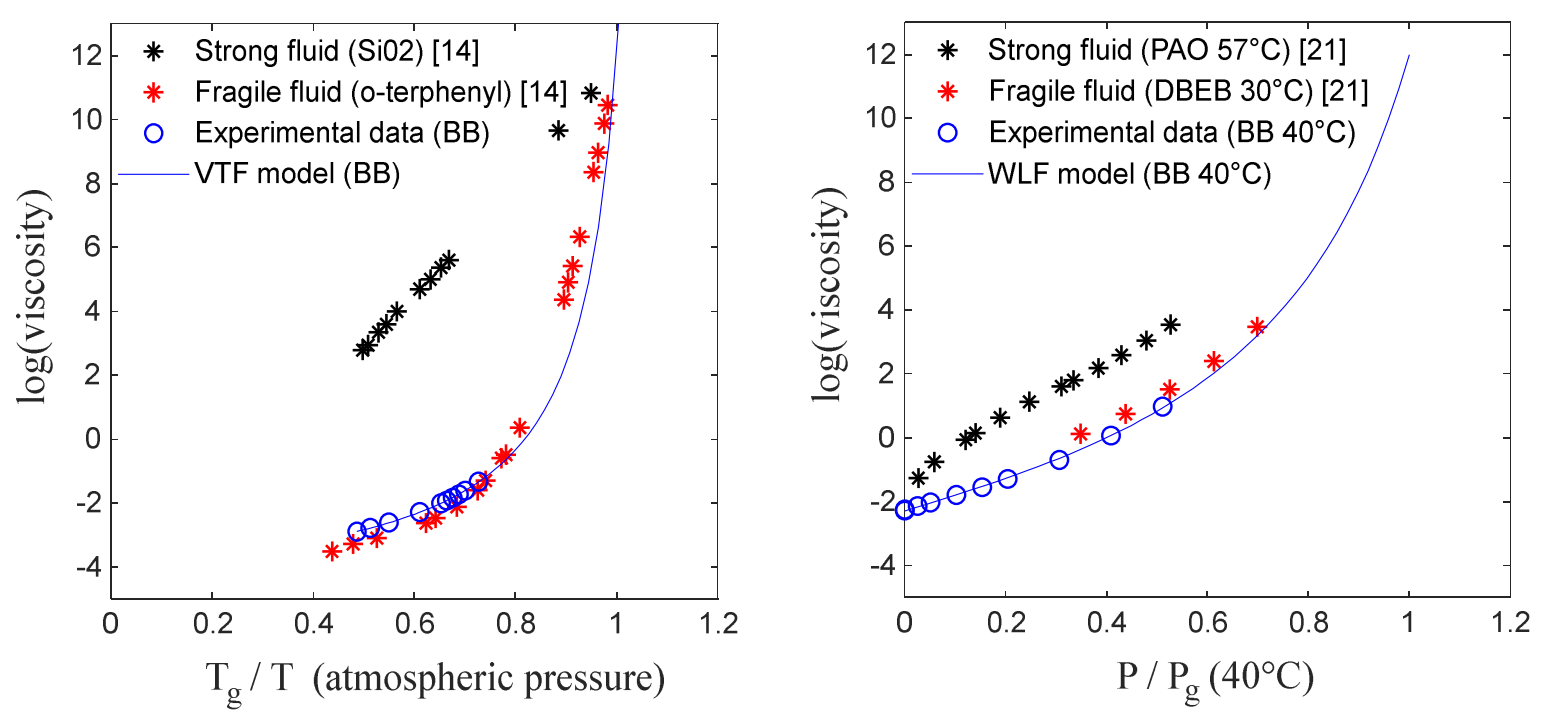

Figure 1: Viscosity (expressed in Pa.s) sensitivity of benzyl benzoate relatively to temperature at atmospheric pressure (left) and to pressure at $40^{\circ} \mathrm{C}$ (right). $\mathrm{T}$ is the temperature of the temperaturecontrolled cell of the rheometer and $\mathrm{P}$ is the hydrostatic pressure in the high pressure device [18].

Reference data for both fragile and strong fluids are from [14] and [21]. 
Figure 1 presents the response of the viscosity of benzyl benzoate relatively to $T$ and $P$. It is clear that, within the experimental domain covered in this work, it follows well the behavior of the two fragile fluids from the literature, meaning that the liquid viscosity is very sensitive to both parameters. The variations predicted by the 2 models employed in this work, namely VTF and WLF (solid lines in Figure 1), are also in good accordance with the results of the literature for both fragile fluids. This agreement remains valid well beyond the experimental domain and confirms the fragile behavior of benzyl benzoate. Comparison with DSC measurements have shown that the extrapolation performed to calculate $T_{g}(0)$ was qualitatively reliable, in the order of a few ${ }^{\circ} \mathrm{C}$ for benzyl benzoate, as for other different lubricants (see [23]).

The modified WLF model was also used to extrapolate for different temperatures the pressures leading to a viscosity of $10^{12} \mathrm{~Pa}$.s, i.e. classically representative in literature of the glass temperature viscosity. Pressures of $0.8,1$ and $1.2 \mathrm{GPa}$ were found at respectively 20,40 and $60^{\circ} \mathrm{C}$. They provide an estimate of the pressures from which the lubricant experiences a transition towards a solid-like behavior. Indeed, the experimental domain was limited to lower values. Nevertheless the very good agreement between rheological measurements and WLF predictions led us to use the model with confidence for higher pressures. Furthermore, the modified WLF model has shown to be very robust relatively to the arbitrary glass transition viscosity of $\eta_{g}=10^{12}$ Pa.s. Indeed, modifying this value (for instance considering $\eta_{g}=10^{8}$ Pa.s as it can be found in the literature) would not significantly modify the glass transition pressure. Finally, Brillouin spectroscopy measurements presented in IV.1 validate these approximate values.

\section{II.2. Diffusion measurements at atmospheric pressure}

Differential scanning calorimetry (DSC) has been performed at atmospheric pressure (under helium atmosphere) from $-130^{\circ} \mathrm{C}$ to $50^{\circ} \mathrm{C}$ at $+10^{\circ} \mathrm{C} / \mathrm{min}$ to validate qualitatively the extrapolation of the VTF model down to the glass transition temperature, and to obtain information on the lubricant physical state over a large range of temperatures. Results are displayed in Figure 2, which superimposes the heat flow (or specific heat) given by DSC and the viscosities both measured in the high pressure falling body viscometer and predicted by the model.

The specific heat exhibits a first steep drop at low temperature which can be explained by a glass transition process. It is nevertheless accompanied by an endothermic peak somehow involving a (partial) phase transition (enthalpy recovery from the glassy state), corresponding to a latent heat of $-2.398 \mathrm{~J} / \mathrm{g}$. The glass transition temperature of $-76.3^{\circ} \mathrm{C}$ derived from DSC measurements (see figure 2) can be compared to $-81.7^{\circ} \mathrm{C}$, obtained by extrapolation with the VTF model (see the vertical dashed line in Figure 2). These values are close to each other, the second one extrapolated from rheological measurements corresponding to the very beginning of the specific heat sudden drop in the DSC experiments (conducted at increasing temperature). The deviation could be inferred to the measurement uncertainties in the two experimental approaches and 
also to the extrapolation at a viscosity of $10^{12} \mathrm{~Pa}$.s which is actually very far from the maximum measured values.

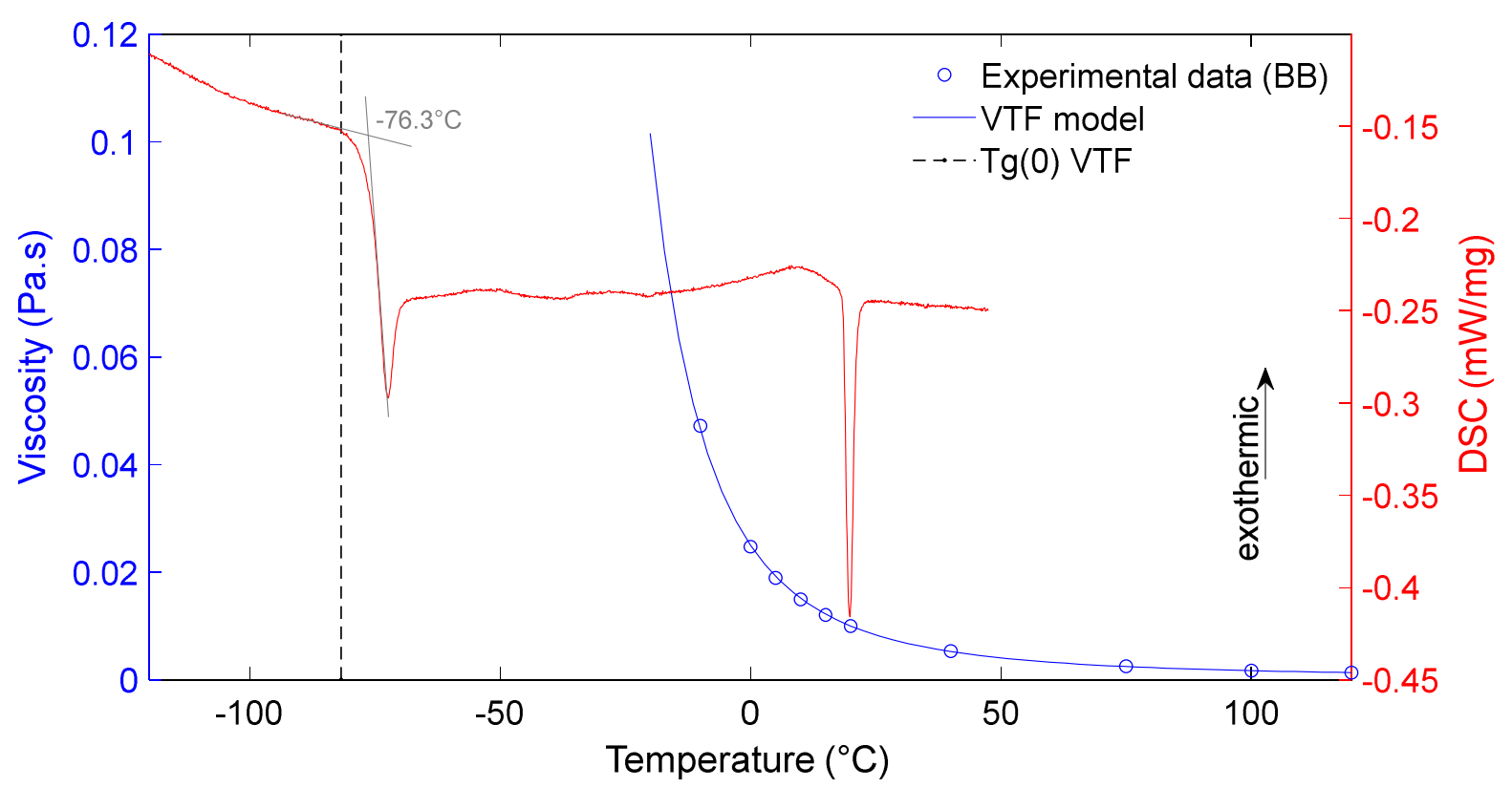

Figure 2: benzyl benzoate viscosity vs temperature (blue curve) measured at atmospheric pressure and fitted to the VTF model (left axis). DSC measurements are superimposed in red (right axis).

A second endothermic peak is observed on DSC measurements at a higher temperature, close to $20^{\circ} \mathrm{C}$, with (almost) no baseline drop. This peak is characteristic of the sample melting from a semi crystalline microstructure to an amorphous one, with a peak thickness representative of the molecules size dispersity, and a latent heat of $-1.933 \mathrm{~J} / \mathrm{g}$ representative of the sample rate of crystallinity. This observation is consistent with the $\mathrm{BB}$ material safety data sheet which indicates a melting point in the range of 18 to $20^{\circ} \mathrm{C}$. Finally, it can be noticed that the viscosity slope strongly softens around this second peak, to reach a weak dependence at higher temperatures.

\section{Experimental approach}

\section{III.1. Brillouin spectroscopy}

Obviously, the transition previously described from free diffusion to (thermally) activated diffusion through cooperative motions and then to a "frozen" state should have consequences on the elastic properties of the material [24-26]. Brillouin light scattering (BLS) measurements have been performed to capture this effect. Following the pioneering work of Dill, Drake and Litovitz [27] who used this technique to establish the viscoelastic properties of a lubricant under high pressure, several studies were conducted to gain more knowledge on the physical state of lubricants at conditions representative of EHD contacts [24-28]. Fluids of 
different chemical natures were investigated, covering the main types of lubricants in use, and aiming at bridging spectroscopic observations with friction [24;28;29] and even with the appearance of the limiting shear stress regime $[28,30]$. Indeed, Brillouin measurements consist in measuring the scattering of an incident light produced by density variations in the sample. These later are induced by the propagation of sound waves, whose propagating velocity is sensitive to the sample elastic properties.

BLS spectra report peaks intensity relatively to the wave frequency, which represents the time between two consecutive waves travelling in the sample and thus which depends on the acoustic wave travelling velocity $c$. The elastic properties of the sample are derived from the inelastic Stokes and Anti Stokes peaks frequencies, located on each side of the Rayleigh elastic scattering peak, expressed in normal incidence as: $\Delta v_{B}=$ $\pm 2 n c / \lambda_{0}$ where $\lambda_{0}$ is the excitation wavelength and $n$ the local refractive index. Therefore, the peaks position depends on the acoustic wave velocity and the refractive index of the sample. A local change of the acoustic wave velocity, driven by a significant increase of the lubricant modulus, is thus expected to shift it. Consequently, a transition from a liquid to a glassy phase should result in a significant shift of the peaks frequency. It can also be noted that the variation of the local refractive index, correlated to the lubricant density, should be negligible in the glassy state. Moreover, density fluctuations dissipate with time, which results in peaks broadening due to the attenuation of the acoustic waves. Hence, the full width at half maximum (FWHM) parameter appears to be the adequate parameter to measure the viscous dissipation in a liquid medium [31].

Brillouin experiments were performed using a Sandercock tandem Fabry-Pérot interferometer [32] coupled with a microscope in back scattering mode. The scattered light was collected by a x10 objective for the 20 and $40^{\circ} \mathrm{C}$ experiments, and a $x 20$ objective for the $60^{\circ} \mathrm{C}$ experiments. The equipment offers an ultimate spectral resolution of $10^{-3} \mathrm{~cm}^{-1}$ and a maximum scanning range, free of overlapping orders, of $50 \mathrm{~cm}^{-1}$. More details on the experimental setup may be found in [32]. A $532 \mathrm{~nm} \mathrm{YAG/} \mathrm{Nd3+} \mathrm{laser} \mathrm{was} \mathrm{used} \mathrm{as} \mathrm{the} \mathrm{light}$ source in normal incidence at a power of approximately $5 \mathrm{~mW}$ measured on the sample [33]. For each isotherm, spectra were recorded during compression and decompression: it was found that the Brillouin signal was completely reversible and reproducible. Spectra were then fitted with a Gaussian function, giving access to peak intensity, peak frequency position and full width at half maximum (FWHM) values.

The sample was confined into a diamond anvil cell (DAC) designed for low-medium pressure experiments (MDAC type BHP For Biology $100^{\circ}$ symmetrical aperture, by Betsa, Nangis, France) for experiments performed at 20 and $40^{\circ} \mathrm{C}$, whereas a double diamond anvils cell was preferred at $60^{\circ} \mathrm{C}$ to reach higher pressures. The sample temperature was adjusted thanks to a heating ring, itself connected to a heating system ensuring a thermal regulation with a precision of $\pm 0.1 \mathrm{~K}$. A calibrated thermocouple was used to measure the temperature close to the sample. Pressure inside the cell was determined from the calibrated shift of the R1 fluorescence line of ruby microspheres added in the sample. In order to ensure both the accuracy of the pressure measurement and the equilibrium state, the shifts of the ruby luminescence peak were measured with a fiber optic portable monochromator (HR Ocean Optics), before and after recording 
each Brillouin spectrum. The spectrometer drift was corrected thanks to a Neon spectral lamp used as a reference to correct the ruby peak position for each spectrum. With all these precautions, the pressure uncertainty inside the DACs was estimated to be of $\pm 50 \mathrm{MPa}$. Acquisition time of 30 minutes per $\mathrm{BB}$ spectrum was required to obtain suitable peak shapes. Brillouin spectra were collected from ambient pressure up to $1.4,1.5$ and $6.5 \mathrm{GPa}$ at 20,40 and $60^{\circ} \mathrm{C}$, respectively.

\section{III.2. Tribological set up}

Friction measurements were performed with a ball-on-disc tribometer described in [2] together with many details on the operating conditions and procedures. Here only the main features are summarized. The bottom of the ball dips into the reservoir containing the lubricant, ensuring fully flooded conditions. The ball, the disc and the lubricant were maintained at constant temperatures of $20,40,60$ and $80^{\circ} \mathrm{C}$ and, at a given temperature, tests were conducted at constant entrainment speed $U_{e}\left(=\left(U_{b a l l}+U_{\text {disc }}\right) / 2\right)$ and for different normal loads and materials combinations. Nominal isothermal conditions were assumed so that the possible influence of thermal heating, analyzed in [2], is considered to be negligible. Moreover, entrainment velocities were adjusted to generate lubricant film thicknesses at the contact center in the range of $110-80 \mathrm{~nm}$ whatever the normal load, due to the weak influence of the latter on this parameter. Combined with the low roughness of the specimen surfaces (in the range of 5-15 $\mathrm{nm}$, see [2]), any possibility of direct contact and thus damage between the contacting surfaces was prevented and tests were carried out in the presence of a full film of lubricant. The applied entrainment velocity ranged from $1.3 \mathrm{~m} / \mathrm{s}$ at $20^{\circ} \mathrm{C}$ to $6.5 \mathrm{~m} / \mathrm{s}$ at $80^{\circ} \mathrm{C}$. The relative uncertainty on the slide-to-roll ratio $S R R=\Delta U / U_{e}$ (with $\Delta U=U_{\text {ball }}-$ $\left.U_{\text {disc }}\right)$ is estimated of $6 \%$ in the worst cases and much lower for the others [2].

The normal load applied on the ball $F_{z}$ and the friction force $F_{x}$ are simultaneously measured through a multiaxis gauge sensor with a maximum uncertainty of $0.82 \%$ and $0.35 \%$ respectively. It leads to a friction coefficient $C F_{x}=F_{x} / F_{z}$ with a relative uncertainty $\Delta C F_{x} / C F_{x}$ of $1.17 \%$. The friction forces obtained at equal absolute values of the slide-to-roll ratio, but with opposite signs, have been systematically averaged. Furthermore, a mean relative standard deviation on $C F_{x}$ of $2.8 \%$ has been derived from some repeated tests, with a rather low dispersion.

Three material configurations were used: a $100 \mathrm{Cr} 6$ bearing steel ball against either a $100 \mathrm{Cr} 6$ steel disc or a sapphire disc, and a tungsten carbide (WC) ball against a WC disc. Different normal loads were applied to reach Hertzian pressures from 0.68 to $3.0 \mathrm{GPa}$, leading to Hertzian contact radii lying between 100 and 290 $\mu \mathrm{m}$.

The constant mean shear stress in the contact was derived from the friction plateau once the relative increase of $C F_{x}$ between two successive values of $S R R$ becomes stable or lower than the experimental uncertainty on CFx (1.17\%): 


$$
\overline{\tau_{L}}=\frac{\max \left(F_{x}\right)}{S}
$$

where $S=\pi a^{2}$ and $a$ is the Hertzian contact radius. It has to be noticed here that the contact area and also the maximum pressure are simply derived from the Hertzian theory. The relative uncertainly on $\overline{\tau_{L}}$ in the whole set of experimentations can be considered lower than $1.2 \%$, obtained from the following error propagation formula:

$$
\frac{\Delta \overline{\tau_{L}}}{\overline{\tau_{L}}}=\frac{\Delta F_{x}}{F_{x}}+\frac{2}{3} \times \frac{\Delta F_{z}}{F_{z}}
$$

\section{III.3. Influence of the contacting bodies}

The use of geometries made of various materials, exhibiting elastic moduli of substantially different values, requires the estimation of the elastic contribution of the solid bodies compared to that of the lubricant deformation in the linear regime of the friction curves. The approach of Bair et al. [34] is applied. It lies in comparing the compliance of both fluid and solids, expressed as the inverse of their linear traction gradients, with the aim to estimate whether the elastic deformation of the solid bodies is likely to influence the friction coefficient evolution at low SRR. The theoretical linear traction gradient due to the solid bodies compliance in a dry contact, given by: $r_{s}=1.78 G / P_{\text {Hertz }}$ [35] with $G$ the equivalent shear modulus calculated from Kalker [36], is compared to the fluid Newtonian traction gradient: $r_{f}=\eta U_{e} /\left(h_{c} \bar{P}\right)$ with $\eta$ the viscosity derived from the linear region of the friction curve.

The ratio $r_{s} / r_{f}$ has been calculated for all the operating conditions of this work. It ranges from 0.8 to $10.7 \%$, suggesting that deformation concentrates in the fluid and that the contribution of the contacting solid bodies in the linear region of the friction curve can be neglected.

\section{Experimental results}

\section{IV.1. Glass transition derived from Brillouin spectroscopy}

The variations of the BB peak frequency and the FWHM parameter, both derived from the Gaussian fit of the Brillouin peaks, exhibited two distinct regimes versus hydrostatic pressure, corresponding to a liquid lubricant and a glassy one. Indeed, in back scattering mode, these parameters are respectively representative of the sample bulk modulus and the attenuation of acoustic longitudinal waves, correlated in the literature to the sample longitudinal viscosity [29]. Basically, high FWHM involve diffusive regimes whereas low FWHM involve propagative ones, with extremely narrow peaks corresponding to solids. These parameters should thus be sensitive to the sample glass transition. Thus, the crossing between the liquid and glassy asymptotic behaviors lead us to identify the glass transition pressure for each of the three temperatures tested. Values of $0.75 \mathrm{GPa}, 1 \mathrm{GPa}$ and $1.2 \mathrm{GPa}$ respectively for $20^{\circ} \mathrm{C}, 40^{\circ} \mathrm{C}$ and $60^{\circ} \mathrm{C}$ were surprisingly in a very good 
agreement with the glass transition pressures derived from the modified WLF Yasutomi model given in section II.1.

The variations of the BB peak frequency and the FWHM parameter are plotted in Figure 3 versus the hydrostatic pressure normalized by the glass transition pressure derived from BLS measurements and for the three temperatures investigated.

Experimental uncertainties have been estimated from the approach proposed in [37]. The experimental noise, defined as the level of fluctuations normalized by the pic intensity (in absolute value), has been calculated for each pressure and temperature condition. It ranges from $17 \mathrm{~dB}$ up to $36 \mathrm{~dB}$, which leads to an experimental accuracy lower than $3 \mathrm{MHz}$. Such an uncertainty is negligible relatively to the orders of magnitude measured. Additionally, uncertainties due to fitting the BLS spectra with a Gaussian function have been derived from the correlation coefficient of each fit. They have been added in Figure 3.
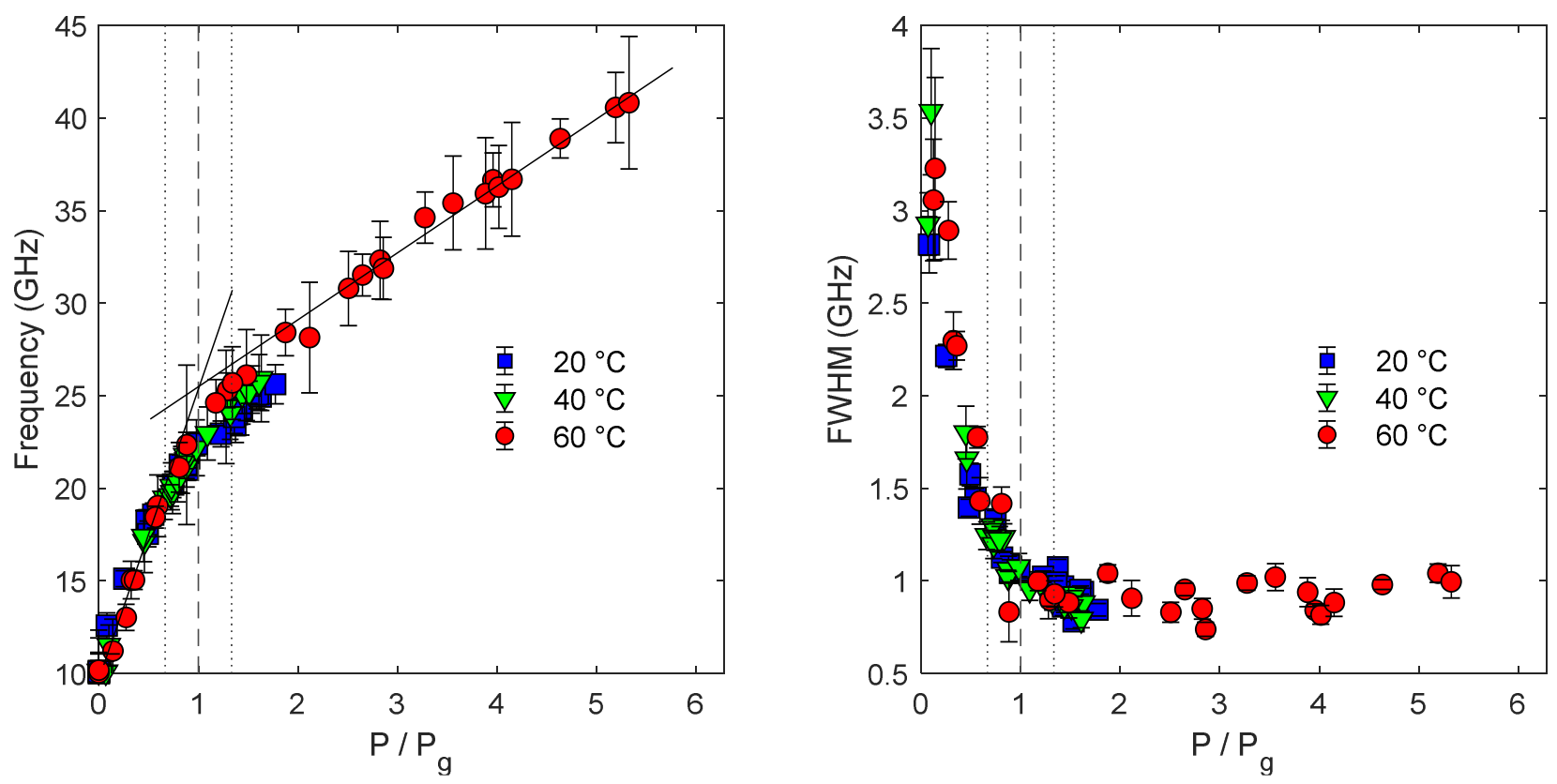

Figure 3: Brillouin spectroscopy results expressed by the peak frequency and the $F W H M$ of BB versus normalized hydrostatic pressure at three temperatures. The dashed vertical lines stand for the transition pressures measured in BLS spectroscopy. The plain lines are just a guide for the eyes. The dotted lines are discussed in sections IV.2 and IV.3.

As shown in Figure 3, and whether it concerns the peak frequency or the full width at half maximum, the spectroscopy data measured for the three temperatures collapse on a single curve when plotted relatively to the normalized pressure. The FWHM exhibits a sharp transition between a first regime during which the FWHM decreases with pressure, and a second regime for which no more variation of the FWHM with this parameter is observed. From viscosity considerations, the first regime can be attributed to the high sensitivity of the sample longitudinal viscosity with pressure in the liquid state, whereas the second regime could be interpreted as the signature of a frozen state of the sample, with no further evolution in viscosity. The low FWHM at high pressures (lower than $1 \mathrm{GHz}$ ) is reminiscent of glassy materials $[38,39]$. This result 
gives more credit to the onset of transition extrapolated from the rheological approach previously presented. These Brillouin spectroscopy results indicate that at the three temperatures of 20,40 and $60^{\circ} \mathrm{C}$ i) benzyl benzoate experiences the same phenomenology of transition, ii) in static condition below the glass transition, the pressure strongly influences the fluid longitudinal viscosity, iii) the fluid longitudinal viscosity appears to be nearly independent of pressure beyond the glass transition pressure.

\section{IV.2. Friction at medium shear rate}

Representative friction curves belonging to the whole set of results are shown on Figure 4, where the pressures mentioned in the legends are the mean pressure values $\left(P_{m}=\frac{2}{3} P_{\text {Hertz }}\right)$. The three first regimes classically identified on a friction curve before the thermal effects become significant can here be distinguished.

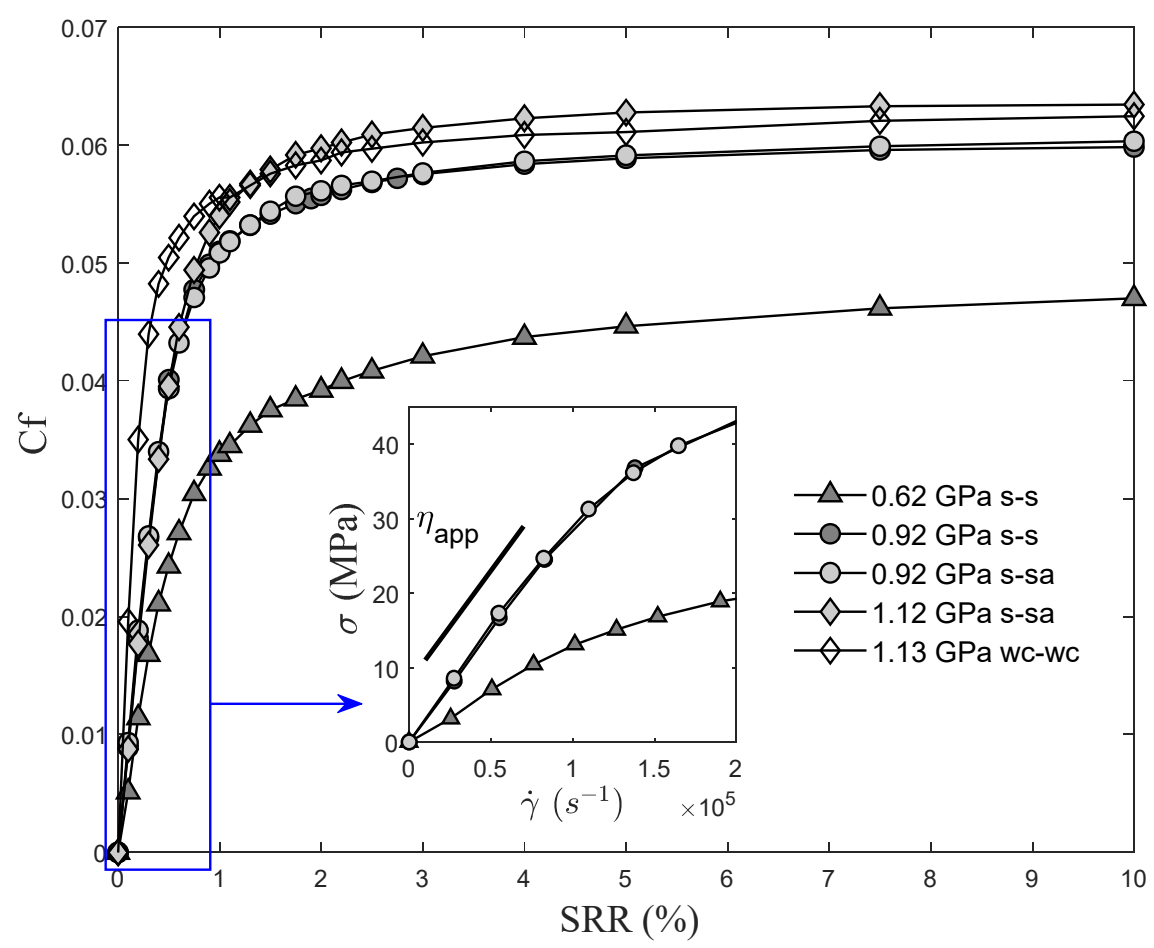

Figure 4: Friction coefficient of benzyl benzoate vs SRR at $40^{\circ} \mathrm{C}$ with steel-steel (s-s), steel-sapphire (s-sa) and tungsten carbide (wc-wc) surfaces. Pressures indicated in the legend correspond to mean contact pressures $\left(\boldsymbol{P}_{m}=\frac{2}{3} \boldsymbol{P}_{\text {Hertz }}\right)$.

Friction curves have then been converted into mean shear stress-mean shear rate charts (see inset in Figure 4) by considering the Hertzian area of contact and the central film thickness $\left(h_{c}\right)$ predicted by the Chittenden expression [40]: the friction force has been divided by the Hertzian area to obtain the mean shear stress and the mean apparent shear rate is the result of $\Delta U / h_{c}$. Finally, the ratio of the mean shear stress to the mean apparent shear rate at very low shear rates has been derived for $|S R R| \leq 0.3 \%$ in order to focus on the first lubricant linear regime, which should not be influenced by the contact sliding. This parameter has a 
dimension of a viscosity. It is then interpreted as a mean apparent viscosity. As shear thinning starts to make the linear regime diverging for SRR beyond a few tenths of a percent, only the first points of each friction curve, aligned with the origin, have been taken into account. The mean apparent viscosities, representative of the physical state of the fluid at the initial stage of the friction tests, are shown in Figure 5 . It should be kept in mind that the non homogeneous distribution of pressure over a contact does not suggest a unique pressure driving friction. Consequently, the mean apparent viscosities are plotted relatively to the mean contact pressure $P_{m}=\frac{2}{3} P_{\text {Hertz }}$ to be directly compared with both rheological and spectroscopy measurements, during which hydrostatic pressures are applied to the whole sample. Data have then been rescaled to compare the results obtained at three temperatures: mean pressure is normalized by the (temperature dependent) glass transition pressure measured in BLS experiements, and mean apparent viscosities are normalized by the viscosity plateau found at the highest pressures applied for each temperature given in Table 2. It should be noted that the plateau viscosity also depends on the contacting bodies materials. Indeed, normalizing by a unique viscosity plateau value per temperature would not lead to a perfect collapse of the curves. Slight variations are noticed between steel and sapphire discs, but higher discrepancies are observed with tungsten carbide. This might be attributed to the different elastic deformations of the solid bodies in elastohydrodynamic conditions. However, it could reasonably be assumed that the materials elasticity effect is a constant term that is suppressed by normalizing the mean apparent viscosity.

\begin{tabular}{|l|c|c|c|}
\hline \multicolumn{1}{|c|}{ Temperature } & $20^{\circ} \mathrm{C}$ & $40^{\circ} \mathrm{C}$ & $60^{\circ} \mathrm{C}$ \\
\hline Materials & 790 & 360 & 145 \\
\hline Steel-Steel & 880 & 360 & 175 \\
\hline Steel-Sapphire & 1610 & 740 & No test \\
\hline WC-WC
\end{tabular}

Table 2: Plateau viscosities (in Pa.s) relatively to temperature and disc materials.

Error bars of $8 \%$ have been added in Figure 5, based on actual uncertainties on $F_{x}, U_{\text {ball }}, U_{\text {disc }}$, on a realistic assumption that the area of the lubricated contacts does not differ from that of the equivalent Hertzian contacts by more than $5 \%$, and an average possible overestimation of $h_{c}$ by $2.5 \%$, according the analysis published by Wheeler et al. [41].

In Figure 5, the relative mean apparent viscosity values corresponding to the three temperatures and the three couples of materials tested overlap onto a single master curve. This suggests that the transition between a liquid-like and a solid-like regime is mainly governed by the thermodynamic state of the material, i.e. by a combination of pressure and temperature dependent parameters. 


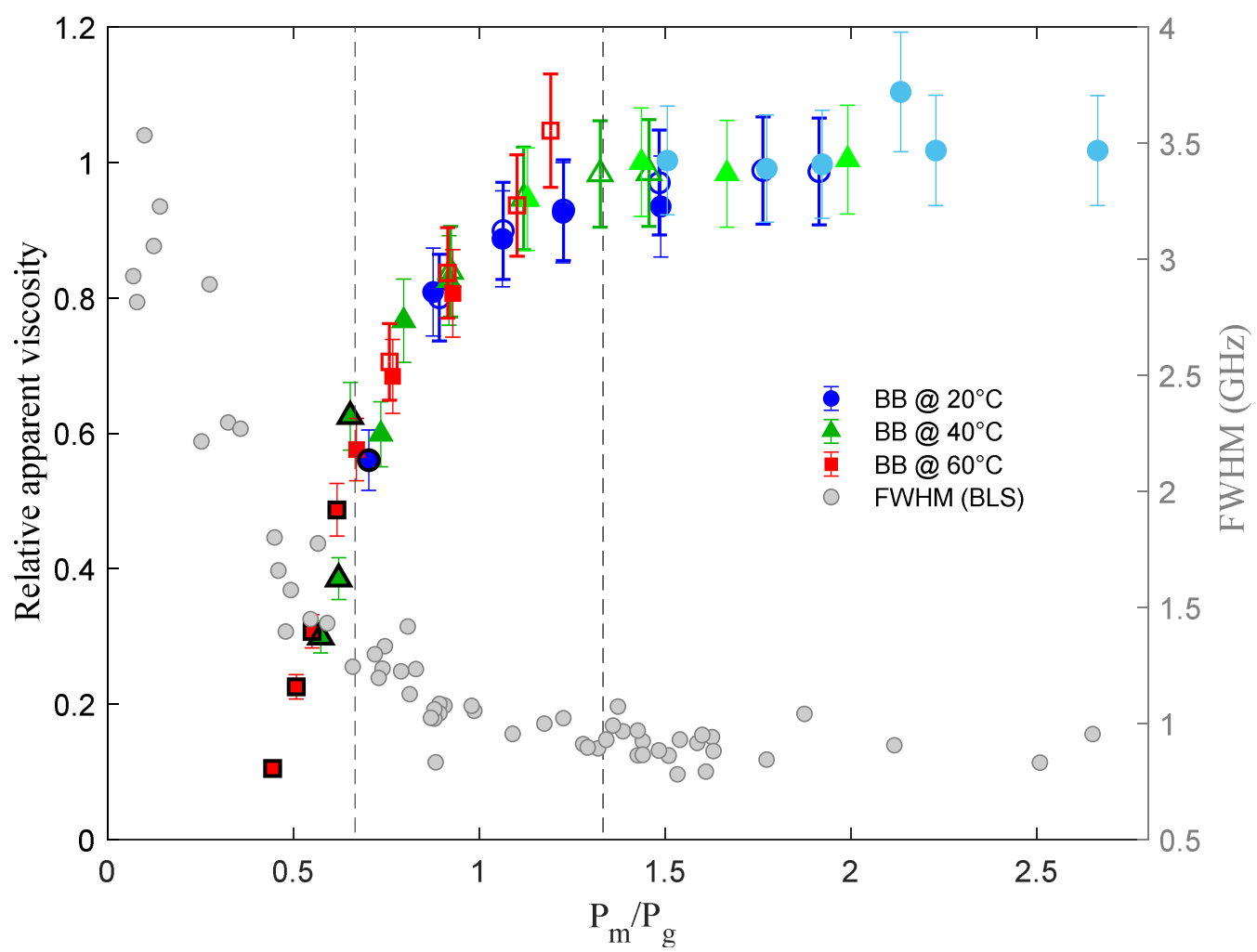

Figure 5: Relative mean apparent viscosity (left axis) of the BB derived from friction measurements at low shear rates vs normalized mean contact pressure, for three temperatures. The vertical dashed lines respectively stand for $\boldsymbol{P}_{\text {Hertz }}=\boldsymbol{P}_{g}$ (left) and $\boldsymbol{P}_{\text {Hertz }}=2 \boldsymbol{P}_{g}$ (right). The black colored circled dots correspond to friction tests with no friction plateau. Plain symbols with dark colors stand for steel-steel surfaces, open symbols stand for steel-sapphire surfaces, and plain symbols with light colors stand for WC-WC surfaces. FWHM values (right axis) of the BB spectra obtained from BLS have been superimposed in light grey.

In this master curve, two asymptotic regimes can be differentiated, depending on the range of mean contact pressure, used here again because of non-constant distribution of P over the contacts area. At low pressure, the mean apparent viscosity increases exponentially with pressure (linear evolution of the curve), which may be associated with a classical piezo-viscous behavior. This evolution extends to a critical pressure from which the exponential trend diverges through a large transition. At higher pressure, the mean apparent viscosity stabilizes to reach a plateau value, becoming almost independent of pressure, consistent with a sample behavior governed mostly by elastic effects for instance. The transition initiates at the first vertical line, standing for $P_{\text {Hertz }}=P_{g}$ or $P_{m}=\frac{2}{3} P_{g}$, with $P_{g}$ the glass transition pressure derived from the BLS measurements, and extends over a large range of pressures around $P_{m}=P_{g}$, up to the second vertical line, standing for $P_{\text {Hertz }}=2 P_{g}$ or $P_{m}=\frac{4}{3} P_{g}$. This strongly suggests that the transition from a viscous to a glassy sample behavior might be responsible for the two asymptotic regimes exhibited on Figure 5 . In addition, this result is reminiscent of the Brillouin spectroscopic FWHM measurements superimposed in this figure. 
Indeed, the range of hydrostatic pressures over which the sample experiences a transition is similar to that of the mean friction pressures, since the two vertical dotted lines reported on Figure 3 respectively correspond to $P / P_{g}=2 / 3$ and $P / P_{g}=4 / 3$. Thus, the progressive nature of the transition may be attributed to the $\mathrm{BB}$ behavior more than the pressure distribution in the ball-on-disc contact.

Furthermore, black surrounded data on Figure 5 stand for friction curves for which the friction plateau criterion given in III.2. was not satisfied, meaning that no plateau regime was detected. It thus appears that the first exponential regime coincides with friction curves that do not satisfy the friction plateau criterion. Conversely, the constant shear stress becomes measurable when the mean apparent viscosity starts experiencing a transition, i.e. for Hertzian pressures higher than the glass transition pressure derived from BLS measurements. Thus, it can reasonably be stated that there is a strong link between the lubricant glass transition and the occurrence of the friction plateau regime in a lubricated contact, even though the nature of this relationship cannot be inferred with confidence from our macroscopic results. However, a better understanding of the macroscopic behavior of the lubricant in a highly loaded contact is still possible from the two vertical lines located at the onset of the viscosity transition and at the beginning of the viscosity plateau. Indeed, the first vertical line $\left(P_{\text {Hertz }}=P_{g}\right)$ shows that, as soon as a very reduced fraction of the lubricant experiences thermodynamic glassy conditions, the macroscopic behavior of the lubricant in the whole contact diverges from a simple exponential pressure-viscosity law, leading to a measurable constant shear stress. This is in line with the conclusions of Bair et al. [42], who showed that i) shear bands develop when the lubricant is subjected to a pressure of the order of its transition pressure, ii) the onset of the first shear band triggers the non-linear behavior of the lubricant. Then, the second vertical line which corresponds to $P_{\text {Hertz }}=2 . P_{g}$, ca. $75 \%$ of the Hertzian contact area is in thermodynamic glassy conditions, according to the Hertzian pressure distribution. This means that the viscosity transition zone develops until $75 \%$ of the contact surface is in a "nominal glassy state". For higher pressures and larger volumes of "nominal glassy" lubricant, the resistance of the contact to the imposed shear no longer varies. It should be noticed that the same experimental approach has been successfully performed with a linear alkane, the squalane, and a commercial turbine mineral oil (Shell T9, see [2]), which led to the same conclusions.

Attention should be paid to the order of magnitude of the viscosity plateaus (cf Table 2), which should correspond to the lubricant in a glassy state. They are, by far, lower than the $10^{12}$ Pa.s empirical criterion used in the modified WLF correlation and used in the literature. Thus, the lubricant physical state in our friction experiments remains unclear. It can be assumed that either the lubricant does not experience a true glass transition (since, for instance, it is not in hydrostatic condition), or the velocity profile is not homogeneous in the contact. Indeed, slip planes or shear localized in areas of lower lubricant viscosity would decrease the tangential force experimentally measured. At this point, the two scenarios cannot be discriminated. However, shear rates considered to derive the mean apparent viscosity, even though only taking into account the lowest SRR values $(0.1-0.3 \%)$ of any friction curve, already reach very high values, 
of order of $5.10^{4}, 10^{5}$ and $2.10^{5} \mathrm{~s}^{-1}$ respectively at 20,40 and $60^{\circ} \mathrm{C}$. As already reported in the literature and discussed in the last part of this paper, the shear imposed to the lubricant can significantly modify the slow dynamics of rearrangements of the bulk molecules.

\section{IV.3. Friction at high shear rate}

At high shear rate, the fluid strongly deforms, which makes difficult to access its physical state. Molecular dynamic simulations revealed that the viscosity scaling does not depend on interface parameters (commensurability between wall and fluid, relative wall orientation, wall stiffness, wall-fluid coupling) or on lubricant chain length [43]. Besides, a striking feature common to all lubricants close to the glass transition and highlighted by many researches is the power-law scaling of the viscosity [8, 43-45]. This scaling $\eta \propto$ $\dot{\gamma}^{-2 / 3}$ is not well understood, but it appears to be a "universal property of lubricants near a glass transition under constant normal load" [43]. More specifically, the exponent of this power law was found to slightly increase in absolute value with pressure, even tending towards unity $[8,43]$.

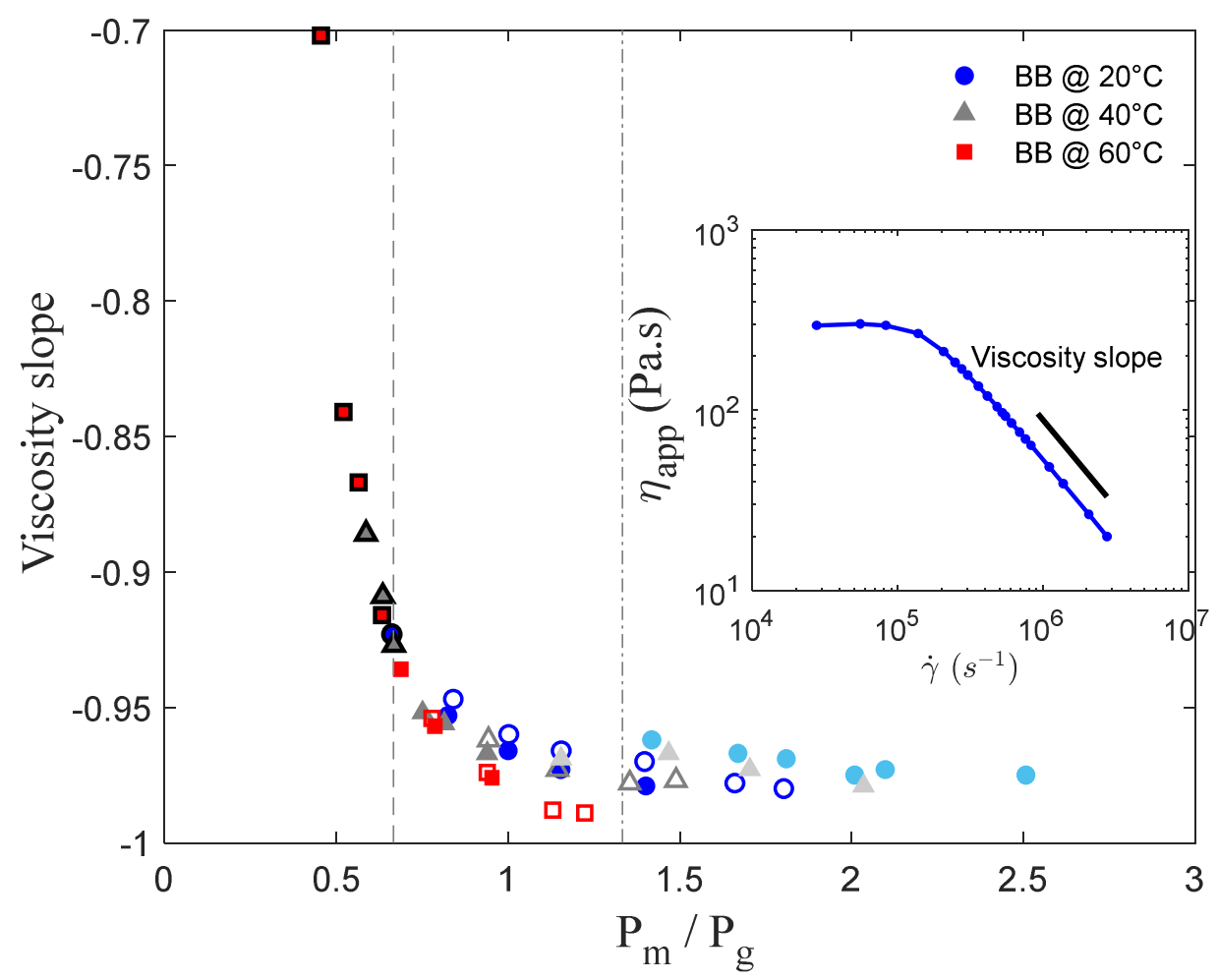

Figure 6: Slope of the mean apparent viscosity of the BB derived from friction measurements at high shear rate vs normalized mean contact pressure, for three temperatures. The vertical dashed lines respectively stand for $\boldsymbol{P}_{\text {Hertz }}=\boldsymbol{P}_{g}$ (left) and $\boldsymbol{P}_{\text {Hertz }}=2 . \boldsymbol{P}_{g}$ (right). The black circled colored dots correspond to friction tests with no friction plateau. Plain symbols with dark colors stand for steel-steel surfaces, open symbols stand for steel-sapphire surfaces, and plain symbols with light colors stand for WC-WC surfaces. Inset: mean apparent viscosity vs shear rate (in log-log scales), showing the viscosity slope extraction. 
Mean apparent viscosities at high SRR have been derived from friction tests by considering the ratio between shear stress and shear rate. The scaling exponents at high shear rates, derived from the power law fit of the last points of friction curves obtained at the highest SRR, are plotted in Figure 6 relatively to the normalized mean contact pressure. They show a similar tendency than the one previously described in Figure 5. Indeed, the exponent first sharply decreases from -0.7 down to -0.92 at the first condition $P_{m} / P_{g}=2 / 3$, indicating a strong sensitivity of BB with pressure. Then, it softens and stabilizes to a mean value of -0.98 from the second condition $P_{m} / P_{g}=4 / 3$. A scaling close to $\eta \propto \dot{\gamma}^{-1}$ was obviously expected at high pressure to match the plateau behavior measured on friction curves and also in agreement with previous studies [8, 43]. However, the comparison of our results with the universal scaling from the literature suggests that the lubricant is already close to the glass transition at the lowest pressure investigated, and that this transition results in an exponent much lower than $-2 / 3$.

\section{Discussion}

The combined analysis of rheological, spectroscopic and friction results revealed a strong correlation between the glass transition experienced by the lubricant and the onset of the plateau-like behavior in friction experiments. Indeed, it has only been observed for a lubricant in a "nominal glassy state". Yet, only moderately large mean apparent viscosities have been derived from these experiments. This could be attributed to either a complex distribution of the shearing across the fluid film thickness or to the occurrence of an intermediate physical state of the fluid, between liquid and glassy. These two scenarios cannot be discarded from macroscopic tests. However, the fluid sensitivity to both pressure and temperature could provide some insights on the most relevant mechanism that may take place within a lubricated contact, involving one of these scenarios.

First, the fragile nature of the lubricant indicates that close to or in a glassy state, it is likely to develop dynamically heterogeneous structures whose characteristic spatial scale extends to a group of molecules. This is confirmed by the Brillouin spectroscopic measurements: significant results have been obtained at typically several tens of $\mathrm{GHz}$. Raman spectroscopy was also performed at several tens of THz on benzyl benzoate confined in a DAC. However, no clear conclusion could be drawn, meaning that the transition experienced by the sample cannot be directly characterized from atomic scale features. In other words, the influence of pressure on the microstructure of BB was successfully probed over the scale of a set of molecules but was imperceptible at the scale of interatomic vibrations. This is also consistent with the description of the glass transition which involves caging processes, strongly slowing down the dynamics of a glassy liquid 
$[7,46]$. One could then wonder whether these dynamical heterogeneous structures involve a transient heterogeneous shear distribution in the contact, macroscopically resulting in the friction plateau. However, recent molecular dynamic simulations [47] evidenced mean shear profiles that are homogeneous in the lubricant film thickness, with no shear localization, neither at the interface nor in the lubricant bulk if atomically rough surfaces are used. As a consequence, one can consider that either i) the dynamics of heterogeneities is faster than the averaging time of velocity profiles (<10 ps) or ii) instantaneous velocity profiles are truly homogeneous and directly come from a homogeneous shear deformation of the glassy lubricant. Due to the high-pressure conditions of the simulations, one can consider that $10 \mathrm{ps}$ is much shorter than the lubricant relaxation time, which makes the first scenario unlikely and validate the second one. This second scenario is in line with the analysis of Debenedetti and coworkers [11] who stated that in a glassy state, molecular rearrangements should be rare and long-lived on the molecular time scale.

The second aspect is related to the physical state of the fluid at the high shear rates at which the friction plateau occurs, between $5.10^{+4}$ and $5.10^{+5} \mathrm{~s}^{-1}$ in our experiments. Indeed, fragile behavior implies that short range molecular order is very sensitive to any energy brought to the system and that the structure is more likely to be "broken" [20-21]. Shear stress could thus strongly affect the short range order structures involved by a glassy state, by altering the high energy barriers responsible for the slowing down of the molecules. This was already noticed by Eyring in 1936 [48] in the case of glassy polymers and was recently raised in [49]. Other works report an increase of molecular mobility induced by the shear softening effect and measured from different experimental devices [50-51]. Thus, due to high shear rates, the lubricant may not experience a true glass transition in the contact, even under nominal glassy state pressure. However it should experience a strong modification of its dynamic behavior, and especially of its $\alpha$ relaxation time (time for molecular rearrangements) while the increase in shear rate decreases the characteristic shearing time $(1 / \dot{\gamma})$. This should participate to the crossover between this characteristic shearing time and the time scale for molecular rearrangements, involving a transition from a viscous regime towards an elasto-plastic-like one. The macroscopic friction should finally result from the competition between the rearrangement processes and the shear rates to which the lubricant is submitted.

\section{Conclusions}

This paper correlates the analysis of rheology, spectroscopy and friction experiments performed on a model lubricant to highlight the influence of the glass transition on the occurrence of a limiting shear stress in highly loaded contacts. Spectroscopic measurements performed at various temperatures and different hydrostatic pressures in a high-pressure diamond anvil cell have proved the existence of a transition associated in the literature with the glass transition. A transition occurred at the same mean pressures in a macroscopic 
contact for all the temperatures tested. Indeed, the mean apparent viscosity derived from the friction force at low sliding followed two asymptotic regimes, located on either side of the transition pressures $P_{g}$ measured by spectroscopy. Furthermore, according to the Hertzian distribution of pressure, the limiting shear stress began to occur in contacts as soon as a very small volume of lubricant reaches a "nominal glassy state". In the light of the results obtained by the different techniques used in this work, it can be said that the friction response in highly loaded contacts, where the pressure is not uniformly distributed, exhibits the following four specific features which appear successively as the pressure increases:

i) For low contact pressures, the lubricant mean apparent viscosity strongly evolves with pressure, as predicted for a liquid in standard conditions. No plateau is then observed on the friction curves.

ii) When increasing the contact normal load, the lubricant experiences a transition as soon as $P_{H e r t z}=$ $P_{g}$, leading to a macroscopic limiting shear stress in the friction curves.

iii) The transition develops up to $P_{H e r t z}=2 . P_{g}$, giving rise to an increase of the relative mean apparent viscosity and a limiting shear stress friction regime.

iv) For $P_{H e r t z}>2 . P_{g}$, no further significant evolution of the lubricant mean apparent viscosity is noticed.

Furthermore, the glass transition pressures have also been extrapolated from a model fitted on the fluid rheological behavior, showing a good agreement between the three different approaches, i.e. rheology, spectroscopy and friction experiments.

However, the high sensitivity with pressure of the lubricant's viscosity and the high shear rates experienced in a contact have raised the question of a true glassy state. The lubricant is more likely to experience an intermediate state, still involving a strong slow-down of the dynamics of molecular rearrangements. Friction plateau would thus be induced, at least partly, by a complex visco - elasto- plastic behavior of the lubricant. A further clarification on the shearing influence on the lubricant dynamics is very likely required to fully explain the friction independence with shear rate.

\section{Acknowledgmements}

The authors are grateful to the SKF company for financial support through the Research Chair "Lubricated Interfaces for the Future" hosted by the INSA de Lyon Foundation. They also want to thank Influtherm (69190 Saint-Fons, France) who kindly provided the DSC analysis. 


\section{Bibliography}

[1] Habchi W., Bair S., Vergne P. : On friction regimes in quantitative elastohydrodynamics. Tribol. Int. 58, 107-117 (2013)

[2] Ndiaye S., Martinie L., Philippon D., Devaux N., Vergne P. : A Quantitative Friction-Based Approach of the Limiting Shear Stress Pressure and Temperature Dependence. Tribol. Lett. 65(4), 149 (2017)

[3] Habchi W., Vergne P., Bair S., Andersson O., Eyheramendy D., Morales-Espejel G.E.: Influence of Pressure and Temperature Dependence of Thermal Properties of a Lubricant on the Behaviour of Circular TEHD Contacts. Tribol. Int. 43(10), 1842-1850 (2010)

[4] Martinie L., Vergne P.: Lubrication at extreme conditions: a discussion about the limiting shear stress concept. Tribol. Lett. 63(2), 21 (2016)

[5] Zhang, Y., Wen, S.: An analysis of elastohydrodynamic lubrication with limiting shear stress: part i-theory and solutions and part ii-load influence. Tribol. Trans. 45(2), 135-144 \& 211-216 (2002)

[6] Johnson, K.L., Tevaarwerk, J.L.: Shear behavior of elastohydrodynamic oil films. Proc. R. Soc. Lond. A 356, 215-236 (1977)

[7] Ediger M.D., Angell C.A., Nagel S.R.: Supercooled liquids and glasses. J. Phys. Chem. 1000, 13200-13212 (1996)

[8] Granick, S.: Motions and relaxations of confined liquids. Science, 253, 1374 (1991)

[9] Angell C.A.: Formation of Glasses from Liquids and Biopolymers. Science 267(5206), 1924-1935 (1995)

[10] Roland C.M.: Characteristic relaxation times and their invariance to thermodynamic conditions. Soft Matter 4, 2316-2322 (2008)

[11] Debenedetti P.G., Stillinger F.H.: Supercooled liquids and the glass transition. Nature 410, 259-267 (2001)

[12] Greaves G.N., Sen S.: Inorganic glasses, glass-forming liquids and amorphizing solids. Advances in Physics 56, 1-166 (2007)

[13] Chang K.: The Nature of Glass Remains Anything but Clear, The New York Times. https://www.nytimes.com/2008/07/29/science/29glass.html. Accessed 29 July 2008

[14] Angell C.A., Ngai K.L., McKenna G.B., McMillan P.F., Martin S.W.: Relaxation in glass forming liquids and amorphous solids: Journal of Applied Physics 88, 3113-3157 (2000)

[15] Roland C.M.: Relaxation Phenomena in Vitrifying Polymers and Molecular Liquids. Macromolecules 43, 7875-7890 (2010)

[16] Salez T., Salez J., Dalnoki-Veress K., Raphaël E., Forrest J.A.: Cooperative strings and glassy interfaces. Proceedings of the National Academy of Sciences 112, 8227-8231 (2015)

[17] Ediger M.D.: Spatially heterogeneous dynamics in supercooled liquids. Annu. Rev. Phys. Chem. 51(1), 99$128(2000)$ 
[18] Mary C., Philippon D., Lafarge L., Laurent D., Rondelez F., Bair S., Vergne P.: New insight into the relationship between molecular effects and the rheological behavior of polymer thickened lubricants under high pressure. Tribol. Lett. 52(3), 357-369 (2013)

[19] Bair S., Mary C., Bouscharain N., Vergne P.: An Improved Yasutomi Correlation for Viscosity at High Pressure. Proc. IMechE, Part J: J. Eng. Tribol. 227(9), 1056-1060 (2013)

[20] Yasutomi S., Bair S., Winer W.O.: An application of a free volume model to lubricant rheology: 1Dependence of viscosity on temperature and pressure. J. Tribol. 106, 291-303 (1984)

[21] Bair S., Roland C.M., Casalini R.: Fragility and the dynamic crossover in lubricants. Proc. IMechE, Part J: J. Eng. Tribol. 221, 801-811 (2007)

[22] Casalini R., Roland C.M.: Why liquids are fragile. Phys. Rev. E 72, 031503 (2005)

[23] Cusseau P., Bouscharain N., Martinie L., Philippon D., Vergne P., Briand F.: Rheological considerations on polymer-based engine lubricants: viscosity index improvers versus thickeners - generalized Newtonian models. Tribol. Trans. 61 (3), 437-447 (2018)

[24] Alsaad M., Bair S., Sanborn D. M., Winer W. O.: Glass transitions in lubricants: its relation to elastohydrodynamic lubrication (EHD). J. Tribol. 100(3), 404-416 (1978)

[25] Harrison G., Trachman E. G.: The role of compressional viscoelasticity in the lubrication of rolling contacts. J. Tribol. 94(4), 306-312 (1972)

[26] Mansot J.L., Martin J.M.: Measurements of local pressure in a static sphere/plane interface containing organic compounds by means of Raman spectroscopy. In Proceedings of the $12^{\text {th }}$ Leeds-Lyon Symposium on Tribology, Lyon (1985). Edited by Dowson D., Taylor C.M., Godet M., Berthe D., Butterworths, London, ISBN 0-408-02960-9, 234-237 (1986)

[27] Dill J.P., Drake P.W., Litovitz T.A.: The Study of Viscoelastic Properties of Lubricants Using High Pressure Optical Techniques. ASLE Trans. 18(3), 202-210, (1975)

[28] Bezot P., Bezot C. H., Berthe D., Dalmaz G., Vergne P.: Viscoelastic parameters of 5P4E as a function of pressure and temperature by light scattering technique. J. Tribol. 108, pp. 579-583 (1986)

[29] Nakamura Y., Fujishiro I., Nishibe K., Kawakami H.: Measurement of physical properties of lubricants under high pressure by Brillouin scattering in a diamond anvil cell. J. Tribol. 117(3), 519-523 (1995)

[30] Fujita Y., Kobayashi H.: Dispersion and attenuation on the Brillouin sound waves of a lubricant: Di(2ethylhexyl) sebacate under high pressures. J. Appl. Phys. 110, 033538 (2011)

[31] Murli M. F. N., Manghnani H., Nellis W. J.: Science and Technology of High Pressure. Proceedings of the International Conference on High Pressure Science and Technology (AIRAPT-17), Honolulu, Hawaii, 25-30 July, Universities Press (1999)

[32] Lindsay S. M., Anderson M. W., Sandercock J. R.: Construction and alignment of a high performance multipass vernier tandem Fabry-Perot interferometer. Rev. Sci. Instrum. 52(10), 1478-1486 (1981) 
[33] Sonneville C., De Ligny D., Mermet A., Champagnon B., Martinet C., Henderson G. H., Deschamps T., Margueritat J., Barthel E.: In situ Brillouin study of sodium alumino silicate glasses under pressure. J. Chem. Phys. 139(7), 74501 (2013)

[34] Bair S., Kotzalas M.: The Contribution of Roller Compliance to Elastohydrodynamic Traction. Tribol. Trans. 49(2), 218-224 (2006)

[35] Johnson K.L., Tevaarwerk J.L.: Shear behavior of elastohydrodynamic oil films. Proc. R. Soc. Lond. A 356, 215-236 (1977)

[36] Kalker J.J.: On the rolling contact of two elastic bodies in the presence of dry friction. PhD Thesis, TU Delft University of Technology (1967)

[37] Margueritat J., Virgone-Carlotta A., Monnier S., Delanoë-Ayari H., Mertani H.C., Berthelot A., Martinet Q., Dagany X., Rivière C., Rieu J.P., Dehoux T.: High-frequency mechanical properties of tumors measured by Brillouin light scattering. Phys. Rev. Lett. 122, 018101 (2019)

[38] Kim M., Besner S., Ramier A., Kwok S.J.J., An J., Scarcelli G., Yun S.H.: Shear Brillouin light scattering microscope. Opt. Express 24, 319-328 (2016)

[39] Jeong M.S., Ko J.H., Ko Y.H., Kim K.J.: High-pressure acoustic properties of glycerol studied by Brillouin spectroscopy. Physica B 478, 27-30 (2015)

[40] Chittenden R.J., Dowson D., Dunn J.F., Taylor C.M.: A Theoretical Analysis of the Isothermal Elastohydrodynamic Lubrication of Concentrated Contacts - Part 2: General Case, with Lubricant Entrainment along Either Principal Axis of the Hertzian Contact Ellipse or at Some Intermediate Angle. Proc. Roy. Soc. London A397, 271-294 (1985)

[41] Wheeler J-D., Vergne P., Fillot N., Philippon D.: On the relevance of analytical film thickness EHD equations for isothermal point contacts: Qualitative or quantitative predictions? Friction 4, 369-379 (2016) [42] Bair S., Qureshi F., Winer W.O.: Observations of shear localization in liquid lubricants under pressure. J. Tribol. 115(3), 507-513 (1993)

[43] Thompson P.A., Grest G.A., Robbins M. O.: Phase Transitions and Universal Dynamics in Confined Films. Phys. Rev. Lett. 68, 3448 (1992)

[44] Hu H.-W., Carson G.A., Granick S.: Relaxation time of confined liquids under shear. Phys. Rev. Lett. 66, 2758-2761 (1991)

[45] Robbins M.O., Smith E.D: Connecting Molecular-Scale and Macroscopic Tribology. Langmuir 12, 45434547 (1996)

[46] Hecksher T., Torchinsky D., Klieber C., Johnson L., Dyre J., Nelson K.A.: Direct test of supercooled liquid scaling relations. arXiv: 1701.01310v1 [cond-mat.soft] (2017)

[47] Porras-Vasquez A., Martinie L., Vergne P., Fillot N.: Independence between friction and velocity distribution in fluids subjected to severe shearing and confinement. Phys. Chem. Chem. Phys. 20, 27280$27293(2018)$ 
[48] Eyring H.: Viscosity, plasticity, and diffusion examples of absolute reaction rates. J. Chem. Phys. 4, 283 (1936)

[49] Kobayashi H., Fujita Y.: Mechanisms for three kinds of limiting shear stresses appearing in the traction modes of viscous, viscoelastic, and glassy states of lubricants. J. Appl. Phys. 115, 223509 (2014)

[50] Loo L.S., Cohen R.E., Gleason K.K.: Chain Mobility in the amorphous region of nylon 6 observed under active uniaxial deformation. Science 288, 116 (2000)

[51] Lee H.-N., Paeng K., Swallen S.F., Ediger M.D.: Direct measurement of molecular mobility in actively deformed polymer glasses. Science 323, 231 (2009) 